\title{
Calculation of the relativistic Bethe logarithm in the two-center problem
}

\author{
Vladimir I. Korobov \\ Bogolyubov Laboratory of Theoretical Physics, Joint Institute for Nuclear Research, Dubna 141980, Russia \\ L. Hilico and J.-Ph. Karr \\ Laboratoire Kastler Brossel, UPMC-Paris-6, ENS, CNRS \\ Case 74, 4 place Jussieu, 75005 Paris, France
}

\begin{abstract}
We present a variational approach to evaluate relativistic corrections of order $\alpha^{2}$ to the Bethe logarithm for the ground electronic state of the Coulomb two center problem. That allows to estimate the radiative contribution at $m \alpha^{7}$ order in molecular-like three-body systems such as hydrogen molecular ions $\mathrm{H}_{2}^{+}$and $\mathrm{HD}^{+}$, or antiprotonic helium atoms. While we get 10 significant digits for the nonrelativistic Bethe logarithm, calculation of the relativistic corrections is much more involved especially for small values of bond length $R$. We were able to achieve a level of 3-4 significant digits starting from $R=0.2 \mathrm{bohr}$, that will allow to reach $10^{-10}$ relative uncertainty on transition frequencies.
\end{abstract}

\section{Introduction}

Considerable effort is currently devoted to high-precision laser spectroscopy of three-body molecular (or moleculelike) systems such as $\mathrm{HD}^{+}\left[1,2, \mathrm{H}_{2}^{+}[3\right.$ and antiprotonic helium [4. These experiments aim at improving the present accuracy of the electron-to-proton and -antiproton mass ratios, for which the uncertainty of spectroscopic data, as well as of theoretical calculations of transition frequencies, should reach a level of about $0.1 \mathrm{ppb}$. Systematic evaluation of leading QED corrections up to the $m \alpha^{6}$ order has improved the theoretical precision in hydrogen molecular ions [5] and antiprotonic helium [6] to $0.3-0.4 \mathrm{ppb}$ and $1 \mathrm{ppb}$, respectively. The main source of theoretical uncertainty is the $m \alpha^{7}$ order one-loop self-energy correction [7, which so far has been evaluated only in hydrogenlike systems. Considering the aimed for accuracy, it is enough to calculate the relativistic Bethe logarithm with 3-4 significant digits and thus it may be obtained in the framework of the adiabatic approximation, i.e. for an electron in the field of two fixed nuclei.

For hydrogen-like ions, the one-loop self-energy contribution to the binding energy of an electron is traditionally expressed as follows [8]

$$
\begin{aligned}
\Delta E_{1-\text { loop }}= & \frac{m_{e} \alpha}{\pi} \frac{(Z \alpha)^{4}}{n^{3}}\left\{\left[A_{41}(n) \ln \left[(Z \alpha)^{-2}\right]+A_{40}(n)\right]+(Z \alpha) A_{50}(n)\right. \\
& \left.+(Z \alpha)^{2}\left[A_{62}(n) \ln ^{2}\left[(Z \alpha)^{-2}\right]+A_{61}(n) \ln \left[(Z \alpha)^{-2}\right]+A_{60}(n)\right]+\ldots\right\} .
\end{aligned}
$$

It is known that among $m \alpha^{5}$ order terms, the Bethe logarithm (which appears in the low-energy part of the nonlogarithmic contribution $A_{40}$ ) is the most difficult quantity for numerical evaluation. Similarly, at the $m \alpha^{7}$ order the low-energy part of $A_{60}$ contains the relativistic Bethe logarithm [9 12] which gives rise to even more severe difficulties. In the present work, we describe a numerical method which allows to obtain these quantities with very good accuracy for a two-center system.

The paper is organized as follows. In Sec. I. we briefly outline the origin of Bethe logarithm contributions in a nonrelativistic quantum electrodynamics (NRQED) approach [13, 14, and give their precise definition. In Sec. II, the asymptotic behavior of the integrands is derived. In Sec. III the numerical method is described in detail, and finally, the nonrelativistic and relativistic Bethe logarithms are calculated for the hydrogen atom, hydrogen molecular ions and antiprotonic helium.

\section{BETHE LOGARITHM: DEFINITIONS}

In this Section natural relativistic units $(\hbar=c=m=1)$ are used, while starting from Sec. II we switch over to the atomic units, which are more suitable for numerical calculations.

As a starting point, we take the nonrelativistic Hamiltonian

$$
H=\frac{\mathbf{p}^{2}}{2 m}+V, \quad V=-\frac{Z_{1} \alpha}{r_{1}}-\frac{Z_{2} \alpha}{r_{2}}
$$

where $r_{1}$ and $r_{2}$ are the distances from the electron to nuclei 1 and 2 , respectively. The case of $Z_{1}=Z_{2}=1$ corresponds to the hydrogen molecular ions and $Z_{1}=2, Z_{2}=-1$ to the antiprotonic helium atom. 


\section{A. The NRQED one-loop self-energy at the $m \alpha^{5}$ order (low photon energy)}

The leading order NRQED interaction with the magnetic field is determined by

$$
H_{I}^{(0)}=-\frac{e}{m} \mathbf{p} \cdot \mathbf{A}-\frac{e}{2 m} \boldsymbol{\sigma} \cdot \mathbf{B}
$$

The first term in this expression is the "dipole" interaction, while the second one is called Fermi's interaction.

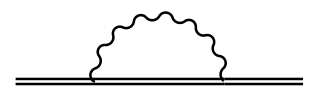

FIG. 1: The NRQED diagram for the leading order one-loop self-energy contribution

It may be shown [15] that the NRQED diagram in Fig. 1 with the Fermi-type interactions on one or both sides of the transverse photon line give vanishing contributions. Thus the low energy part contribution, which stems from the NRQED diagram in Fig. 1 with two dipole vertices may be expressed:

$$
E_{L-r e t}=\frac{\alpha^{3}}{4 \pi^{2} m^{2}} \int_{|\mathbf{k}|<\Lambda} \frac{d^{3} k}{k}\left(\delta^{i j}-\frac{k^{i} k^{j}}{k^{2}}\right)\left\langle e^{i \mathbf{k r}_{a}} \mathbf{p}\left(\frac{1}{E_{0}-H-k}\right) \mathbf{p} e^{-i \mathbf{k r}_{b}}\right\rangle-\delta m\left\langle\psi_{0} \mid \psi_{0}\right\rangle
$$

where $\delta m$ is a "mass renormalization" term. Here and throughout this paper it is assumed that in $\langle\ldots\rangle$ on the left and right-hand sides of brackets stands $\psi_{0}$, a stationary Schrödinger eigenstate of the Hamiltonian operator of Eq. 22, if not otherwise stated.

To get the leading $m \alpha^{5}$ order contribution, one neglects retardation, replacing the exponential factors in 3 by unity, which leads to the nonrelativistic dipole approximation

$$
E_{L 0}=\frac{2 \alpha^{3}}{3 \pi m^{2}} \int_{0}^{\Lambda} k d k\left\langle\mathbf{p}\left(\frac{1}{E_{0}-H-k}\right) \mathbf{p}\right\rangle-\delta m\left\langle\psi_{0} \mid \psi_{0}\right\rangle=\frac{2 \alpha^{3}}{3 \pi m^{2}} \int_{0}^{\Lambda} k d k P_{n d}(k)-\delta m\left\langle\psi_{0} \mid \psi_{0}\right\rangle
$$

with

$$
P_{n d}(k)=\left\langle\mathbf{p}\left(E_{0}-H-k\right)^{-1} \mathbf{p}\right\rangle
$$

The integral in (4) contains a linearly divergent term which corresponds to the electron's mass renormalization, as was shown by Bethe in 1947 [16]. It also contains a logarithmic term, where the dependance on the cutoff parameter $\Lambda$ is canceled by the logarithmic contribution from the high-energy part [17]. After these two terms are dropped out, the remaining nonlogarithmic contribution at order $m \alpha^{5}$ may be written $\left(E_{h}=m \alpha^{2}\right.$ is the Hartree energy)

$$
\mathcal{N}(n ; R)=\int_{0}^{E_{h}} k d k\left\langle\mathbf{p}\left(\frac{1}{E_{0}-H-k}+\frac{1}{k}\right) \mathbf{p}\right\rangle+\int_{E_{h}}^{\infty} \frac{d k}{k}\left\langle\mathbf{p} \frac{\left(E_{0}-H\right)^{2}}{E_{0}-H-k} \mathbf{p}\right\rangle
$$

and determines the numerator of the Bethe logarithm, while the denominator is expressed by

$$
\mathcal{D}(n ; R)=\frac{1}{2}\langle\Delta V\rangle
$$

The Bethe logarithm itself is defined as the ratio

$$
\beta_{n r}(n ; R)=\frac{\mathcal{N}}{\mathcal{D}} .
$$

Here $n$ denotes a set of state quantum numbers.

\section{B. One-loop self-energy contributions at the $m \alpha^{7}$ order}

Here, for convenience of reading, we keep the notation of [12] wherever possible. There are three types of relativistic corrections to the leading order expression (4), which give a contribution at order $m \alpha^{7}$ : 


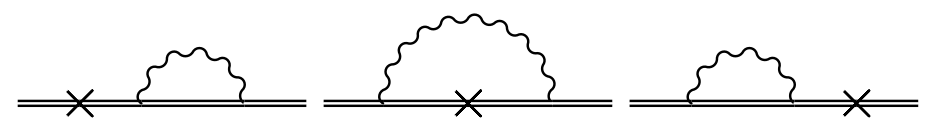

FIG. 2: NRQED diagrams for relativistic corrections to the electron line, which contribute to self-energy at order $m \alpha^{7}$.

1. Relativistic corrections due to the Breit-Pauli interaction (Fig. 2)

$$
E_{L 1}=\frac{2 \alpha^{3}}{3 \pi m^{2}} \int_{0}^{\Lambda} k d k \delta_{H_{B}}\left\langle\mathbf{p}\left(\frac{1}{E_{0}-H-k}\right) \mathbf{p}\right\rangle=\frac{2 \alpha^{3}}{3 \pi m^{2}} \int_{0}^{\Lambda} k d k P_{r c}^{(1)}(k)
$$

where

$$
\begin{gathered}
P_{r c}^{(1)}(k)=\delta_{H_{B}}\left\langle\mathbf{p}\left(\frac{1}{E_{0}-H-k}\right) \mathbf{p}\right\rangle \equiv 2\left\langle H_{B} Q\left(E_{0}-H\right)^{-1} Q \mathbf{p}\left(E_{0}-H-k\right)^{-1} \mathbf{p}\right\rangle \\
+\left\langle\mathbf{p}\left(E_{0}-H-k\right)^{-1}\left(H_{B}-\left\langle H_{B}\right\rangle\right)\left(E_{0}-H-k\right)^{-1} \mathbf{p}\right\rangle .
\end{gathered}
$$

Here $Q$ is a projection operator: $Q=I-\left|\psi_{0}\right\rangle\left\langle\psi_{0}\right|$. Eq. (10) represents the third order term in the Rayleigh-Schrödinger perturbation theory. The relativistic Breit-Pauli Hamiltonian for the two-center problem is expressed

$$
H_{B}=-\frac{\mathbf{p}^{4}}{8 m^{3}}+\frac{1}{8 m^{2}}\left[4 \pi Z_{1} \delta\left(\mathbf{r}_{1}\right)+4 \pi Z_{2} \delta\left(\mathbf{r}_{2}\right)\right],
$$

the spin interaction is neglected.

For reasons which will be discussed later, it is convenient to split $P_{r c}^{(1)}$ into two parts:

$$
\begin{aligned}
& P_{r c}^{(1 a)}(k)=\left\langle\mathbf{p}\left(E_{0}-H-k\right)^{-1}\left(H_{B}-\left\langle H_{B}\right\rangle\right)\left(E_{0}-H-k\right)^{-1} \mathbf{p}\right\rangle \\
& P_{r c}^{(1 b)}(k)=2\left\langle H_{B} Q\left(E_{0}-H\right)^{-1} Q \mathbf{p}\left(E_{0}-H-k\right)^{-1} \mathbf{p}\right\rangle .
\end{aligned}
$$

2. Modification of vertex interactions in the self-energy diagram

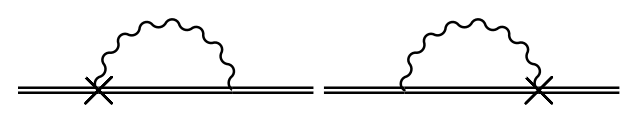

FIG. 3: The NRQED diagrams for the self-energy with modified vertex interactions at order $m \alpha^{7}$

The next order NRQED interactions $\left(\sim \alpha^{2} H_{I}^{(0)}\right)$ with a magnetic field are determined by [14, 18,

$$
H_{I}^{(2)}=\frac{e}{2 m^{3}} p^{2} \mathbf{p} \cdot \mathbf{A}+\frac{e^{2}}{4 m^{2}} \sigma^{i j}\left(\nabla^{j} V\right) A^{i},
$$

thus modifying vertex functions as shown on the diagrams in Fig. (3); one gets

$$
E_{L 3}=\frac{4 \alpha^{3}}{3 \pi m^{2}} \int_{0}^{\Lambda} k d k\left\langle\delta \mathbf{J}\left(\frac{1}{E_{0}-H-k}\right) \mathbf{p}\right\rangle=\frac{4 \alpha^{3}}{3 \pi m^{2}} \int_{0}^{\Lambda} k d k P_{r c}^{(2)}(k)
$$

where $\delta J^{i}=-p^{2} p^{i}-\frac{1}{2} \sigma^{i j} \nabla^{j} V$, and

$$
P_{r c}^{(2)}(k)=\left\langle\left(-p^{2} p^{i}-\frac{1}{2} \sigma^{i j} \nabla^{j} V\right)\left(E_{0}-H-k\right)^{-1} p^{i}\right\rangle .
$$

3. It remains to consider the effect of retardation (see Eq. (3p). We obtain the nonrelativistic quadrupole contribution, which results from the Taylor series expansion of $e^{i(\mathbf{k r})}=1+i(\mathbf{k r})-(\mathbf{k r})^{2} / 2+\ldots$

$$
E_{L 2}=\frac{2 \alpha^{3}}{3 \pi m^{2}} \int_{0}^{\Lambda} k d k P_{n q}(k)
$$




$$
\begin{aligned}
P_{n q}(k)=\frac{3 k^{2}}{8 \pi} \int_{S} d \Omega_{\mathbf{n}}\left(\delta^{i j}-n^{i} n^{j}\right) & \left\{\left\langle p^{i}(\mathbf{n} \cdot \mathbf{r})\left(E_{0}-H-k\right)^{-1}(\mathbf{n} \cdot \mathbf{r}) p^{i}\right\rangle\right. \\
& \left.-\left\langle p^{i}(\mathbf{n} \cdot \mathbf{r})^{2}\left(E_{0}-H-k\right)^{-1} p^{i}\right\rangle\right\},
\end{aligned}
$$

where $\mathbf{k}=k \mathbf{n}$.

Similarly to the nonrelativistic Bethe logarithm considered above, the relativistic Bethe logarithm corresponds to the finite part of the integrals (9), (12) and (14), i.e. divergent terms in $\Lambda$ must be subtracted [12]. It is thus essential to study the asymptotic behavior of the integrands in the $k \rightarrow \infty$ limit.

\section{ASYMPTOTIC BEHAVIOUR OF THE INTEGRANDS AT $k \rightarrow \infty$}

Our approach to obtain asymptotic expansions stems from ideas first formulated by C. Schwartz in [19. The first step is to note that in expression (4) the integrand's form is that of a second-order perturbation correction. It may be calculated via the first-order perturbation wave function $\psi_{1}$, which can be obtained by solving the differential equation

$$
\left(E_{0}-H-k\right) \psi_{1}=\nabla \psi_{0}
$$

and then one calculates the integrand by evaluating

$$
P_{n d}(k)=\left\langle\psi_{0}|\nabla| \psi_{1}\right\rangle \text {. }
$$

\section{A. Nonrelativistic Bethe logarithm}

The first order nonrelativistic perturbation wave function for $k \rightarrow \infty$ to a good extent may be approximated (see [19, 20]) by

$$
\psi_{1}(\mathbf{r}) \approx \frac{1}{k}\left[\frac{Z_{1} \mathbf{r}_{1}}{r_{1}}+\frac{Z_{2} \mathbf{r}_{2}}{r_{2}}\right] \psi_{0}(r)-\frac{1}{k^{2}}\left\{\frac{Z_{1} \mathbf{r}_{1}}{r_{1}^{3}}\left[1-e^{-\mu r_{1}}\left(1+\mu r_{1}\right)\right]+\frac{Z_{2} \mathbf{r}_{2}}{r_{2}^{3}}\left[1-e^{-\mu r_{2}}\left(1+\mu r_{2}\right)\right]\right\} \psi_{0}(r),
$$

here $\mu=\sqrt{2 k}$. This function has a proper behaviour at $r \rightarrow 0$.

By substituting (18) into expression $\frac{1}{k}\left\langle\nabla^{2}\right\rangle-\frac{1}{k}\left\langle\psi_{0}|[H, \nabla]| \psi_{1}\right\rangle$ (see for details [19, 20]) one gets for the nonrelativistic dipole term

$$
\begin{aligned}
P_{n d}(k)=\frac{1}{k}\left\langle\nabla^{2}\right\rangle & +\frac{1}{2 k^{2}}\langle\Delta V\rangle \\
& -\frac{1}{k^{3}}\left\{\left[Z_{1}^{2} \sqrt{2 k}-Z_{1}^{3} \ln k\right] 4 \pi\left\langle\delta\left(\mathbf{r}_{1}\right)\right\rangle+\left[Z_{2}^{2} \sqrt{2 k}-Z_{2}^{3} \ln k\right] 4 \pi\left\langle\delta\left(\mathbf{r}_{2}\right)\right\rangle\right\}+\ldots
\end{aligned}
$$

in which all terms (including the last one in $\ln k / k^{3}$ ) are correct. For higher-order terms in $1 / k$ we use the same expansion as for the hydrogen atom

$$
\sum_{m=1}^{M} \frac{Q_{1 m}^{n d} \sqrt{k}+Q_{2 m}^{n d} \ln k+Q_{3 m}^{n d}}{k^{m+3}},
$$

which is in the latter case known analytically [21] (See also [9]). 


\section{B. Relativistic Bethe logarithm}

Substituting again the wave function $\psi_{1}$ from $(18)$ into the matrix elements which appear in the integrands $P_{i}(k)$ in Eqs. 15, 11a, 11b and (13), one gets

$$
\begin{aligned}
& P_{n q}(k)=-\frac{1}{2}\left\langle\nabla^{2}\right\rangle-\frac{1}{k}\left[\frac{\left\langle\nabla^{4}\right\rangle}{5}+2 \pi\left(Z_{1}\left\langle\delta\left(\mathbf{r}_{1}\right)\right\rangle+Z_{2}\left\langle\delta\left(\mathbf{r}_{2}\right)\right\rangle\right)\right] \\
& +\frac{2 Z_{1}^{2}\left(\sqrt{2 k}-Z_{1} \ln k\right)}{k^{2}} 4 \pi\left\langle\delta\left(\mathbf{r}_{1}\right)\right\rangle+\frac{2 Z_{2}^{2}\left(\sqrt{2 k}-Z_{2} \ln k\right)}{k^{2}} 4 \pi\left\langle\delta\left(\mathbf{r}_{2}\right)\right\rangle+\ldots \\
& =F_{n q}+\frac{A_{n q}}{k}+\frac{B_{n q}}{k^{3 / 2}}+\frac{C_{n q} \ln k}{k^{2}}+\frac{D_{n q}}{k^{2}}+\ldots \\
& P_{r c}^{(1 a)}(k)=-\frac{Z_{1}^{2} \sqrt{2 k}}{4 k^{2}} 4 \pi\left\langle\delta\left(\mathbf{r}_{1}\right)\right\rangle-\frac{Z_{2}^{2} \sqrt{2 k}}{4 k^{2}} 4 \pi\left\langle\delta\left(\mathbf{r}_{2}\right)\right\rangle+\ldots \\
& =\frac{B_{r c}^{(1 a)}}{k^{3 / 2}}+\frac{D_{r c}^{(1 a)}}{k^{2}}+\ldots \\
& P_{r c}^{(1 b)}(k)=\frac{2}{k}\left\langle\left(H_{B}-\left\langle H_{B}\right\rangle\right)\left(E_{0}-H\right)^{-1} \nabla^{2}\right\rangle \\
& +\frac{Z_{1}^{2}\left(2 \sqrt{2 k}+Z_{1} \ln k\right)}{4 k^{2}} 4 \pi\left\langle\delta\left(\mathbf{r}_{1}\right)\right\rangle+\frac{Z_{2}^{2}\left(2 \sqrt{2 k}+Z_{2} \ln k\right)}{4 k^{2}} 4 \pi\left\langle\delta\left(\mathbf{r}_{2}\right)\right\rangle+\ldots \\
& =\frac{A_{r c}^{(1 b)}}{k}+\frac{B_{r c}^{(1 b)}}{k^{3 / 2}}+\frac{C_{r c}^{(1 b)} \ln k}{k^{2}}+\frac{D_{r c}^{(1 b)}}{k^{2}}+\ldots \\
& P_{r c}^{(2)}(k)=\frac{\left\langle p^{4}\right\rangle}{k}+\frac{Z_{1}^{2}\left(-\sqrt{8 k}+Z_{1} \ln k\right)}{k^{2}} 4 \pi\left\langle\delta\left(\mathbf{r}_{1}\right)\right\rangle+\frac{Z_{2}^{2}\left(-\sqrt{8 k}+Z_{2} \ln k\right)}{k^{2}} 4 \pi\left\langle\delta\left(\mathbf{r}_{2}\right)\right\rangle+\ldots \\
& =\frac{A_{r c}^{(2)}}{k}+\frac{B_{r c}^{(2)}}{k^{3 / 2}}+\frac{C_{r c}^{(2)} \ln k}{k^{2}}+\frac{D_{r c}^{(2)}}{k^{2}}+\ldots
\end{aligned}
$$

For higher order terms, in the case of $P_{n q}, P_{r c}^{(1 a)}$ and $P_{r c}^{(2)}$ the form of the asymptotic expansion is found to be similar to the nonrelativistic Bethe logarithm (Eq. 20), for example:

$$
P_{r c}^{(1 a)}(k)-\frac{B_{r c}^{(1 a)}}{k^{3 / 2}}-\frac{D_{r c}^{(1 a)}}{k^{2}}=\sum_{m=1}^{M} \frac{Q_{1 m}^{(1 a)} \sqrt{k}+Q_{2 m}^{(1 a)} \ln k+Q_{3 m}^{(1 a)}}{k^{m+2}}
$$

with equivalent expressions for $P_{n q}(k)$ and $P_{r c}^{(2)}(k)$. The $P_{r c}^{(1 b)}$ term has an essentially different asymptotic behavior:

$$
P_{r c}^{(1 b)}(k)-\frac{A_{r c}^{(1 b)}}{k}-\frac{B_{r c}^{(1 b)}}{k^{3 / 2}}-\frac{C_{r c}^{(1 b)} \ln k}{k^{2}}-\frac{D_{r c}^{(1 b)}}{k^{2}}=\frac{1}{k^{2}} \sum_{m=1}^{M} \sum_{n=0}^{m} \frac{S_{m n}^{(1 b)} \ln ^{n} k}{k^{m / 2}}
$$

This is one of the reasons why the $P_{r c}^{(1)}$ term has been separated in two contributions.

In actual calculations coefficients of the asymptotic expansion $A, B, C$, and $F$ are calculated from expectation values of the operators appearing in the Eqs. $21 \mathrm{a})-21 \mathrm{~d})$, while the unknown coefficients $D, Q$, and $S$ are obtained by fitting of the numerically evaluated integrand using Eqs. 22, -23 .

\section{Final expression of the relativistic Bethe Logarithm}

In view of the asymptotic expansion obtained in the previous paragraph, the relativistic Bethe logarithm, which is given by the finite part of the integrals [9]-14, can be written as follows:

$$
\mathcal{L}=\beta_{1}^{(a)}+\beta_{1}^{(b)}+\beta_{2}+\beta_{3}
$$




$$
\begin{aligned}
& \beta_{1}^{(a)}=\frac{2}{3} \int_{0}^{E_{h}} k d k\left[P_{r c}^{(1 a)}(k)-\frac{B_{r c}^{(1 a)}}{k^{3 / 2}}\right] \\
& +\frac{2}{3} \int_{E_{h}}^{\infty} k d k\left[P_{r c}^{(1 a)}(k)-\frac{B_{r c}^{(1 a)}}{k^{3 / 2}}-\frac{D_{r c}^{(1 a)}}{k^{2}}\right] \\
& \beta_{1}^{(b)}=\frac{2}{3} \int_{0}^{E_{h}} k d k\left[P_{r c}^{(1 b)}(k)-\frac{A_{r c}^{(1 b)}}{k}-\frac{B_{r c}^{(1 b)}}{k^{3 / 2}}\right] \\
& +\frac{2}{3} \int_{E_{h}}^{\infty} k d k\left[P_{r c}^{(1 b)}(k)-\frac{A_{r c}^{(1 b)}}{k}-\frac{B_{r c}^{(1 b)}}{k^{3 / 2}}-\frac{C_{r c}^{(1 b)} \ln k}{k^{2}}-\frac{D_{r c}^{(1 b)}}{k^{2}}\right] \\
& \beta_{2}=\frac{4}{3} \int_{0}^{E_{h}} k d k\left[P_{n q}(k)-F_{n q}-\frac{A_{n q}}{k}-\frac{B_{n q}}{k^{3 / 2}}\right] \\
& +\frac{4}{3} \int_{E_{h}}^{\infty} k d k\left[P_{n q}(k)-F_{n q}-\frac{A_{n q}}{k}-\frac{B_{n q}}{k^{3 / 2}}-\frac{C_{n q} \ln k}{k^{2}}-\frac{D_{n q}}{k^{2}}\right] \\
& \beta_{3}=\frac{2}{3} \int_{0}^{E_{h}} k d k\left[P_{r c}^{(2)}(k)-\frac{A_{r c}^{(2)}}{k}-\frac{B_{r c}^{(2)}}{k^{3 / 2}}\right] \\
& +\frac{2}{3} \int_{E_{h}}^{\infty} k d k\left[P_{r c}^{(2)}(k)-\frac{A_{r c}^{(2)}}{k}-\frac{B_{r c}^{(2)}}{k^{3 / 2}}-\frac{C_{r c}^{(2)} \ln k}{k^{2}}-\frac{D_{r c}^{(2)}}{k^{2}}\right] .
\end{aligned}
$$

Here the terms which are subtracted from the first line of each equation appear in the expansions due to a formal Taylor series expansion in powers of $(Z \alpha)^{2}$ of the QED one-loop self-energy correction, which is expressed [8]:

$$
\Delta E=-i \frac{e^{2}}{(2 \pi)^{4}} \int \frac{d^{4} k}{k^{2}+i \epsilon}\left\langle\bar{\psi}_{0}^{D}(\mathbf{r})\left|e^{i \mathbf{k r}} \gamma_{\mu} S_{F}\left(\mathbf{r}, \mathbf{r}^{\prime}, E_{0}-k^{0}\right) \gamma^{\mu} e^{-i \mathbf{k r} \mathbf{r}^{\prime}}\right| \psi_{0}^{D}\left(\mathbf{r}^{\prime}\right)\right\rangle-\delta m\left\langle\bar{\psi}_{0}^{D} \mid \psi_{0}^{D}\right\rangle
$$

here $S_{F}\left(\mathbf{r}, \mathbf{r}^{\prime}, E\right)$ is the Dirac-Coulomb propagator and $\psi_{0}^{D}$ is the Dirac wave function. These extra terms do not appear in asymptotic expansion of the integrand in Eq. 25) and should be withdrawn.

Thus our definition coincides with that of [12] (see prescriptions after Eq. (3.16) in that Ref.).

\section{NUMERICAL SCHEME}

Here we present a numerical scheme to evaluate the Bethe logarithm (8) and its relativistic corrections (24) for the two-center Coulomb problem.

\section{A. Variational expansion}

A variational expansion for the electronic wave function is taken in the form $\left(Z_{1} \neq Z_{2}\right)[22$ :

$$
\Psi_{m}(\mathbf{r})=e^{i m \varphi} r^{|m|} \sum_{i=1}^{\infty} C_{i} e^{-\alpha_{i} r_{1}-\beta_{i} r_{2}},
$$

where $r$ is a distance from the electron to the $z$-axis and

$$
r=\frac{1}{2 R} \sqrt{2 r_{1}^{2} r_{2}^{2}+2 r_{1}^{2} R^{2}+2 r_{2}^{2} R^{2}-r_{1}^{4}-r_{2}^{4}-R^{4}} .
$$

For $Z_{1}=Z_{2}$ the variational wave function should be symmetrized

$$
\Psi\left(\mathbf{r}_{1}, \mathbf{r}_{2}\right)=e^{i m \varphi} r^{|m|} \sum_{i=1}^{\infty} C_{i}\left(e^{-\alpha_{i} r_{1}-\beta_{i} r_{2}} \pm e^{-\beta_{i} r_{1}-\alpha_{i} r_{2}}\right)
$$



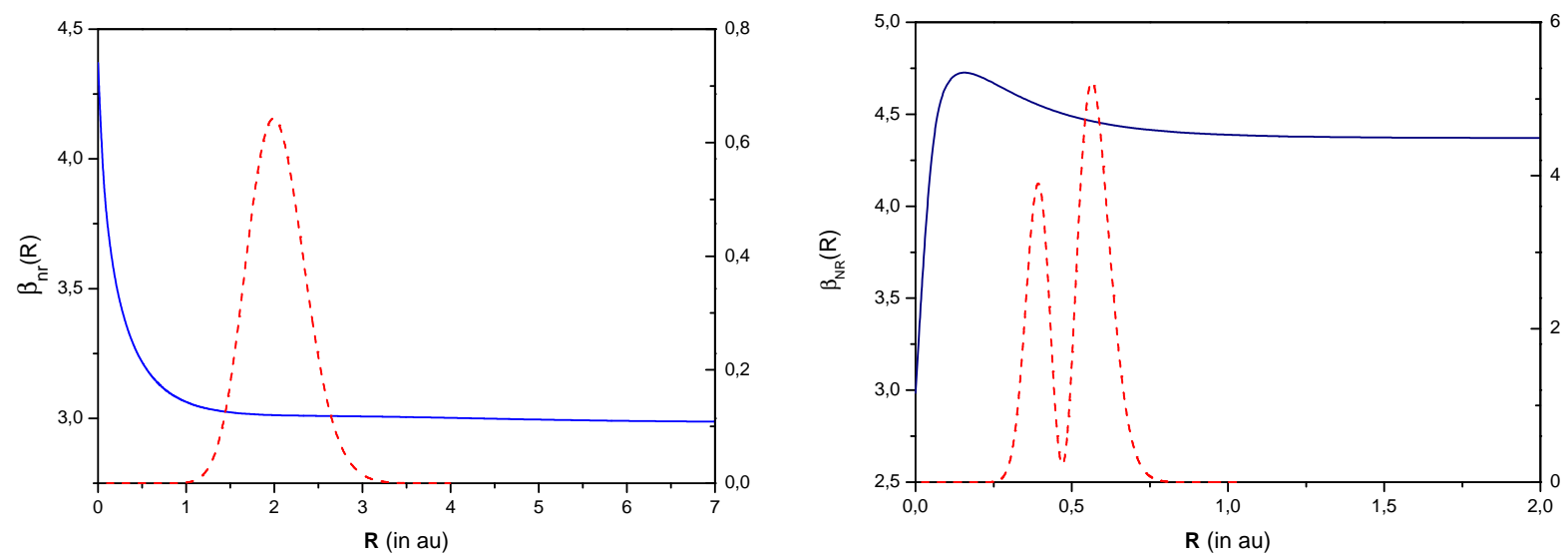

FIG. 4: The nonrelativistic Bethe logarithm for the ground $(1 s \sigma)$ electronic state for $Z_{1}=Z_{2}=1$ (left) and $Z_{1}=2, Z_{2}=-1$ (right). Dashed lines show the vibrational wave functions for the ground state in $\mathrm{H}_{2}^{+}$ion (left) and the $(36,34)$ state in ${ }^{4} \mathrm{He}^{+} \bar{p}$ (right).

where $(+)$ is used to get a gerade electronic state and $(-)$ is for an ungerade state, respectively. Parameters $\alpha_{i}$ and $\beta_{i}$ are generated in a quasi-random manner [23]

$$
\alpha_{i}=\left\lfloor\frac{1}{2} i(i+1) \sqrt{p_{\alpha}}\right\rfloor\left(A_{2}-A_{1}\right)+A_{1} .
$$

Here $\lfloor x\rfloor$ designates the fractional part of $x, p_{\alpha}$ is a prime number, and $\left[A_{1}, A_{2}\right]$ is a real variational interval, which has to be optimized. Parameters $\beta_{i}$ are obtained in a similar way. Details may be found elsewhere 22, 24.

All the integrands in Eqs. (5), (15), 11a), 11b) and (13) have the form of a second-order perturbation expression, i.e. they involve an operator $\left(E_{0}-H-k\right)^{-1}$. We thus diagonalize the matrix of the Hamiltonian for intermediate states to get a set of (pseudo)state wavefunctions $\psi_{m}$ and energies $E_{m}$. A similar approach was used to compute the nonrelativistic Bethe logarithm for the three-body Coulomb problem in [20].

The basis for intermediate states is constructed as follows.

(1) We use a regular basis set (with regular values of the exponents $\alpha, \beta$ ), similar to that used for the initial state.

(2) We build a special basis set with exponentially growing parameters for $r_{1}$ :

$$
\begin{cases}A_{1}^{(0)}=A_{1}, & A_{2}^{(0)}=A_{2}, \\ A_{1}^{(n)}=\tau^{n} A_{1}, & A_{2}^{(n)}=\tau^{n} A_{2},\end{cases}
$$

where $\tau=A_{2} / A_{1}$. Typically $\left[A_{1}, A_{2}\right]=[2.5,4.5]$, and $n_{\max }=5-7$, which corresponds to the photon energy interval $k \in\left[0,10^{4}\right]$.

(3) We add a similar basis set for $r_{2}$. Note that this last step may be omitted in the case $Z_{1}=Z_{2}$ where the basis is symmetrized.

\section{B. Nonrelativistic Bethe logarithm}

After expansion on the basis for intermediate state, the expression of the integrand becomes

$$
P_{n d}(k)=\sum_{m} \frac{\left\langle\psi_{0}|\boldsymbol{\nabla}| \psi_{m}\right\rangle^{2}}{E_{0}-E_{m}-k},
$$

so the integral appearing in the low-energy part of the numerator is

$$
\int_{0}^{\Lambda} k d k P_{n d}(k)=\sum_{m}\left\langle\psi_{0}|\nabla| \psi_{m}\right\rangle^{2}\left[\Lambda-\left(E_{0}-E_{m}\right) \ln \left|\frac{E_{0}-E_{m}}{E_{0}-E_{m}-\Lambda}\right|\right] .
$$



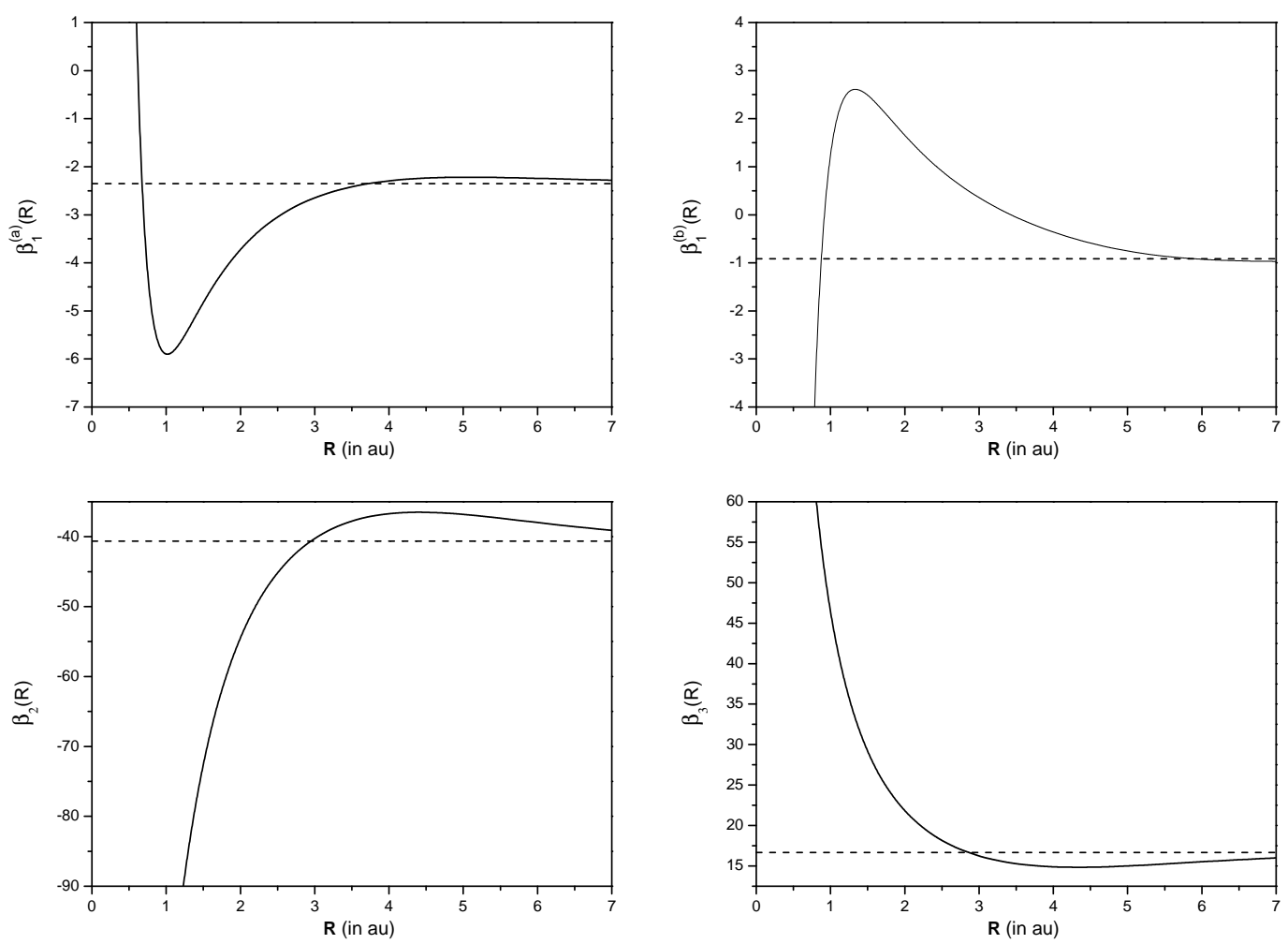

FIG. 5: The four contributions to the relativistic Bethe logarithm for the ground $\left(1 s \sigma_{g}\right)$ electronic state, for $Z_{1}=Z_{2}=1$. Dashed lines show their respective values for the 1s state of a hydrogen atom 12 .

It remains to calculate the matrix elements of the impulse operator. Its standard components are

$$
\begin{aligned}
& \nabla_{0}^{(1)}=\nabla_{z} \\
& \nabla_{ \pm 1}^{(1)}=\mp \frac{1}{\sqrt{2}}\left(\nabla_{x} \pm i \nabla_{y}\right)
\end{aligned}
$$

Assuming, from now on, that $\psi_{0}$ is a $\sigma$-state, action on $\psi_{0}$ of the impulse operator may be expressed as follows

$$
\begin{aligned}
& \nabla_{0}^{(1)} \psi_{0}=\left[\left(z+\frac{R}{2}\right) \frac{1}{r_{1}} \partial_{r_{1}}+\left(z-\frac{R}{2}\right) \frac{1}{r_{2}} \partial_{r_{2}}\right] \psi_{0}, \\
& \nabla_{ \pm 1}^{(1)} \psi_{0}=r e^{ \pm i \varphi}\left(\frac{1}{r_{1}} \partial_{r_{1}}+\frac{1}{r_{2}} \partial_{r_{2}}\right) \psi_{0} .
\end{aligned}
$$

Here $z=\left(r_{1}^{2}-r_{2}^{2}\right) /(2 R)$. Using these relations then calculation of the matrix elements is straightforward [22, 24].

\section{Relativistic corrections to the Bethe logarithm}

We devote one paragraph to each term of Eq. 24), giving additional details which are necessary for their numerical evaluation. Note that the terms $\beta_{1}^{(a)}$ and $\beta_{1}^{(b)}$ are treated numerically in an independent way, which is a further reason for separating these two contributions.

1. For $\beta_{1}^{(a)}$, the integrand can be written as

$$
P_{r c}^{(1 a)}(k)=\left\langle\psi_{1}\left|\left(H_{B}-\left\langle H_{B}\right\rangle\right)\right| \psi_{1}\right\rangle .
$$

To evaluate $\left\langle\psi_{1}\left|\mathbf{p}^{4}\right| \psi_{1}\right\rangle$, it is convenient to use the following identity (see Eq. [16)

$$
\mathbf{p}^{2} \psi_{1}=2\left[\left(E_{0}-V-k\right) \psi_{1}-\nabla \psi_{0}\right] .
$$



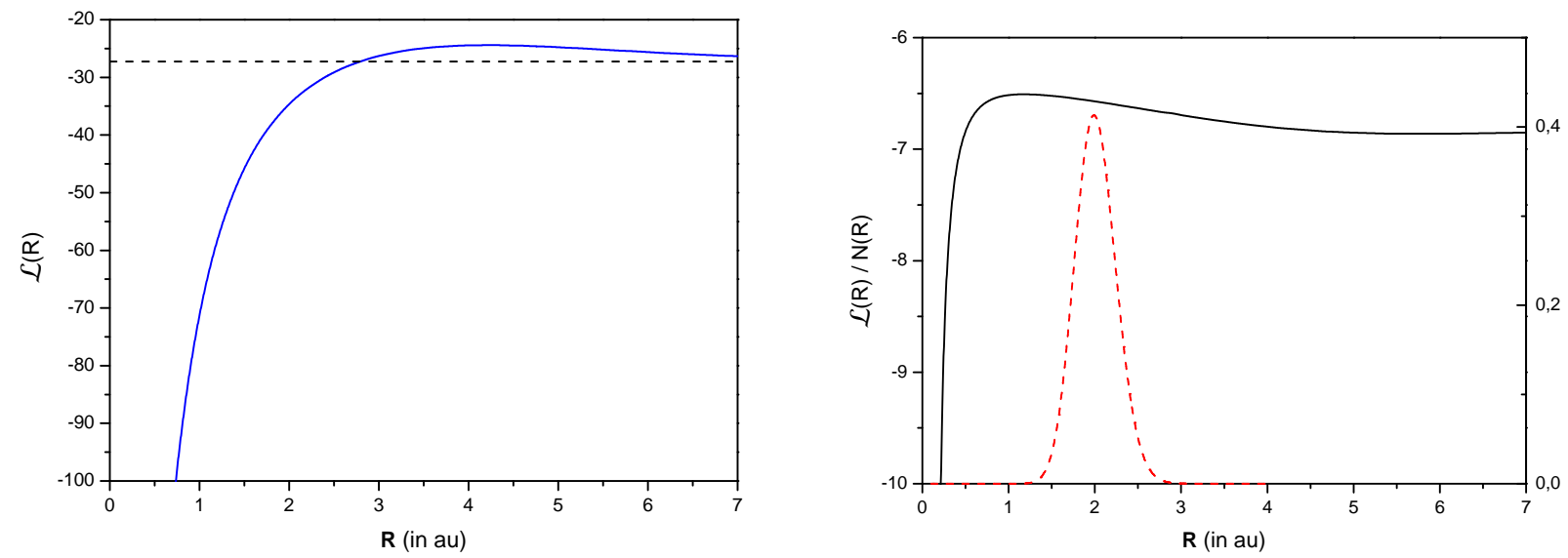

FIG. 6: (Left) The relativistic Bethe logarithm for the ground $\left(1 s \sigma_{g}\right)$ electronic state, for $Z_{1}=Z_{2}=1$. The dashed line is the $\mathcal{L}(1 s)$ for the 1 s state of a hydrogen atom 12. (Right) The same data, but normalized by the delta function distribution: $N(R)=4 \pi\left(Z_{1}^{3} \delta\left(\mathbf{r}_{1}\right)+Z_{2}^{3} \delta\left(\mathbf{r}_{2}\right)\right)$. The dashed line is the vibrational wave function for the ground state of $\mathrm{H}_{2}^{+}$.

Then

$$
\left\langle\psi_{1}\left|\mathbf{p}^{4}\right| \psi_{1}\right\rangle=4\left\langle\psi_{1}\left|\left(E_{0}-V-k\right)^{2}\right| \psi_{1}\right\rangle-4\left\langle\psi_{1}\left|\left(E_{0}-V-k\right)\right| \psi_{0}\right\rangle+\left\langle\nabla^{2}\right\rangle
$$

and, for arbitrary $k, \psi_{1}(k)$ may be expressed:

$$
\psi_{1}(k)=\sum_{m} \frac{\left|\psi_{m}\right\rangle\left\langle\psi_{m}|\nabla| \psi_{0}\right\rangle}{E_{0}-E_{m}-k}
$$

2. In order to get $P_{r c}^{(1 b)}(k)$ we first solve the equation

$$
\left(E_{0}-H\right) \psi_{B}=\left(H_{B}-\left\langle H_{B}\right\rangle\right) \psi_{0}
$$

It can be shown that $\psi_{B}$ behaves at small $r_{1}\left(\right.$ or $\left.r_{2}\right)$ as

$$
\psi_{B}\left(r_{1}, r_{2}\right)=\left(\frac{Z_{1}}{4 r_{1}}-\frac{Z_{1}^{2}}{2} \ln r_{1}\right) \psi_{0}\left(r_{1}, r_{2}\right)+\left(\frac{Z_{2}}{4 r_{2}}-\frac{Z_{2}^{2}}{2} \ln r_{2}\right) \psi_{0}\left(r_{1}, r_{2}\right)+\tilde{\psi}_{B}\left(r_{1}, r_{2}\right)
$$

where $\tilde{\psi}_{B}\left(r_{1}, r_{2}\right)$ is a regular function. Then equation for $\tilde{\psi}_{B}\left(r_{1}, r_{2}\right)$ may be written

$$
\left(E_{0}-H\right) \tilde{\psi}_{B}=\left(H_{B}-\left\langle H_{B}\right\rangle\right) \psi_{0}+\left[H,\left(\frac{Z_{1}}{4 r_{1}}+\frac{Z_{2}}{4 r_{2}}-\frac{Z_{1}^{2}}{2} \ln r_{1}-\frac{Z_{2}^{2}}{2} \ln r_{2}\right)\right] \psi_{0}
$$

Thus substituting $\psi_{B}\left(r_{1}, r_{2}\right)$ from Eq. (39) into Eq. (11b), one gets

$$
\begin{aligned}
P_{r c}^{(1 b)}(k)=2\langle & \left.\left(\frac{Z_{1}}{4 r_{1}}+\frac{Z_{2}}{4 r_{2}}-\frac{Z_{1}^{2}}{2} \ln r_{1}-\frac{Z_{2}^{2}}{2} \ln r_{2}\right) Q \mathbf{p}\left(E_{0}-H-k\right)^{-1} \mathbf{p}\right\rangle \\
& +2\left\langle\tilde{\psi}_{B}\left|Q \mathbf{p}\left(E_{0}-H-k\right)^{-1} \mathbf{p}\right| \psi_{0}\right\rangle
\end{aligned}
$$

The derivation of matrix elements involving logarithms may be found in the Appendix.

3. For the evaluation of $P_{n q}(k)$, Eq. (15) must be transformed to separate contributions from operators of different ranks. Denoting $A_{s}=\left(E_{0}-H-k\right)^{-1}$,

$$
P_{n q}(k)=\frac{3}{8 \pi} \int d \Omega_{\mathbf{n}}\left(\delta^{i j}-n^{i} n^{j}\right) n^{l} n^{m} \int k^{3} d k\left\{\left\langle p^{i} r^{l} A_{s} r^{m} p^{j}\right\rangle-\left\langle p^{i} r^{l} r^{m} A_{s} p^{j}\right\rangle\right\}
$$




\begin{tabular}{|c|c|c|c|c|c|c|}
\hline$R$ & $\beta_{1}^{(a)}$ & $\beta_{1}^{(b)}$ & $\beta_{2}$ & $\beta_{3}$ & $\mathcal{L}$ & $\mathcal{L} / N(R)$ \\
\hline 0.2 & 199.124 & -362.792 & -718.74 & 500.9 & -381.5 & -9.4774 \\
\hline 0.3 & 71.425 & -155.864 & -408.41 & 244.09 & -248.76 & -7.7149 \\
\hline 0.4 & 26.866 & -73.891 & -293.55 & 153.814 & -186.76 & -7.0981 \\
\hline 0.5 & 8.5247 & -36.4368 & -232.469 & 111.218 & -149.163 & -6.8191 \\
\hline 0.6 & 0.3419 & -17.8754 & -192.985 & 86.9905 & -123.529 & -6.6763 \\
\hline 0.7 & -3.4173 & -8.19603 & -164.670 & 71.3995 & -104.884 & -6.5980 \\
\hline 0.8 & -5.1044 & -2.99381 & -143.164 & 60.5041 & -90.7581 & -6.5534 \\
\hline 0.9 & -5.7669 & -0.16341 & -126.255 & 52.4484 & -79.7371 & -6.5273 \\
\hline 1.0 & -5.94144 & 1.36042 & -112.653 & 46.2540 & -70.9802 & -6.5145 \\
\hline 1.1 & -5.85242 & 2.14699 & -101.528 & 41.3527 & -63.8807 & -6.5084 \\
\hline 1.2 & -5.64166 & 2.50981 & -92.3095 & 37.3908 & -58.0506 & -6.5071 \\
\hline 1.3 & -5.37875 & 2.62712 & -84.5938 & 34.1346 & -53.2109 & -6.5097 \\
\hline 1.4 & -5.10067 & 2.60269 & -78.0750 & 31.4227 & -49.1503 & -6.5145 \\
\hline 1.5 & -4.82677 & 2.49792 & -72.5270 & 29.1400 & -45.7159 & -6.5212 \\
\hline 1.6 & -4.56684 & 2.34961 & -67.7744 & 27.2013 & -42.7903 & -6.5294 \\
\hline 1.7 & -4.32531 & 2.17994 & -63.6799 & 25.5425 & -40.2828 & -6.5387 \\
\hline 1.8 & -4.10371 & 2.00222 & -60.1351 & 24.1145 & -38.1221 & -6.5489 \\
\hline 1.9 & -3.90197 & 1.82441 & -57.0531 & 22.8786 & -36.2520 & -6.5598 \\
\hline 2.0 & -3.71921 & 1.65108 & -54.3637 & 21.8045 & -34.6274 & -6.5712 \\
\hline 2.1 & -3.55413 & 1.48479 & -52.0099 & 20.8676 & -33.2117 & -6.5831 \\
\hline 2.2 & -3.40532 & 1.32679 & -49.9446 & 20.0480 & -31.9752 & -6.5953 \\
\hline 2.3 & -3.27133 & 1.17755 & -48.1290 & 19.3294 & -30.8933 & -6.6077 \\
\hline 2.4 & -3.15078 & 1.03708 & -46.5306 & 18.6985 & -29.9458 & -6.6203 \\
\hline 2.5 & -3.04237 & 0.90509 & -45.1223 & 18.1441 & -29.1155 & -6.6329 \\
\hline 2.6 & -2.94493 & 0.78117 & -43.8811 & 17.6566 & -28.3883 & -6.6456 \\
\hline 2.7 & -2.85740 & 0.66481 & -42.7875 & 17.2283 & -27.7518 & -6.6583 \\
\hline 2.8 & -2.77881 & 0.55550 & -41.8248 & 16.8522 & -27.1958 & -6.6709 \\
\hline 2.9 & -2.70829 & 0.45272 & -40.9786 & 16.5527 & -26.6814 & -6.6758 \\
\hline 3.0 & -2.64510 & 0.35594 & -40.2365 & 16.2347 & -26.2909 & -6.6955 \\
\hline 3.2 & -2.53796 & 0.17881 & -39.0229 & 15.7664 & -25.6156 & -6.7192 \\
\hline 3.4 & -2.45271 & 0.02095 & -38.1126 & 15.4188 & -25.1256 & -6.7416 \\
\hline 3.6 & -2.38557 & -0.12013 & -37.4498 & 15.1695 & -24.7860 & -6.7624 \\
\hline 3.8 & -2.33346 & -0.24634 & -36.9899 & 15.0008 & -24.5689 & -6.7815 \\
\hline 4.0 & -2.29385 & -0.35913 & -36.6963 & 14.8980 & -24.4513 & -6.7985 \\
\hline 4.2 & -2.26461 & -0.45961 & -36.5387 & 14.8490 & -24.4139 & -6.8135 \\
\hline 4.4 & -2.24395 & -0.54861 & -36.4913 & 14.8435 & -24.4404 & -6.8264 \\
\hline 4.6 & -2.23034 & -0.62682 & -36.5320 & 14.8726 & -24.5165 & -6.8370 \\
\hline 4.8 & -2.22246 & -0.69487 & -36.6417 & 14.9288 & -24.6303 & -6.8456 \\
\hline 5.0 & -2.21917 & -0.75336 & -36.8039 & 15.0053 & -24.7712 & -6.8523 \\
\hline
\end{tabular}

TABLE I: Relativistic Bethe logarithm for the ground $\left(1 s \sigma_{g}\right)$ electronic state, for $Z_{1}=Z_{2}=1$.

and one obtains

$$
\begin{aligned}
& \frac{3}{8 \pi} \int d \Omega_{\mathbf{n}}\left(\delta^{i j}-n^{i} n^{j}\right) n^{l} n^{m} \int k^{3} d k\left\langle p^{i} r^{l} A_{s} r^{m} p^{j}\right\rangle \\
&= \int k^{3} d k\left\{\frac{3}{10}\left\langle\left[S_{i j}^{(2)}\right]^{\dagger} A_{s} S_{i j}^{(2)}\right\rangle-\frac{1}{4}\left\langle[\mathbf{p} \times \mathbf{r}] A_{s}[\mathbf{r} \times \mathbf{p}]\right\rangle\right\} \\
&= \int k^{3} d k\left\{\frac{9}{20}\left\langle\left[S_{\mu}^{(2)}\right]^{\dagger} A_{s} S_{\mu}^{(2)}\right\rangle-\frac{1}{4}\left\langle[\mathbf{p} \times \mathbf{r}] A_{s}[\mathbf{r} \times \mathbf{p}]\right\rangle\right\} \\
& \frac{3}{8 \pi} \int d \Omega_{\mathbf{n}}\left(\delta^{i j}-n^{i} n^{j}\right) n^{l} n^{m} \int k^{3} d k\left\langle p^{i} r^{l} r^{m} A_{s} p^{j}\right\rangle \\
&=\int k^{3} d k\left\{\frac{2}{5}\left\langle p^{i} r^{2} A_{s} p^{i}\right\rangle-\frac{1}{5}\left\langle(\mathbf{p r}) r^{j} A_{s} p^{j}\right\rangle\right\}
\end{aligned}
$$



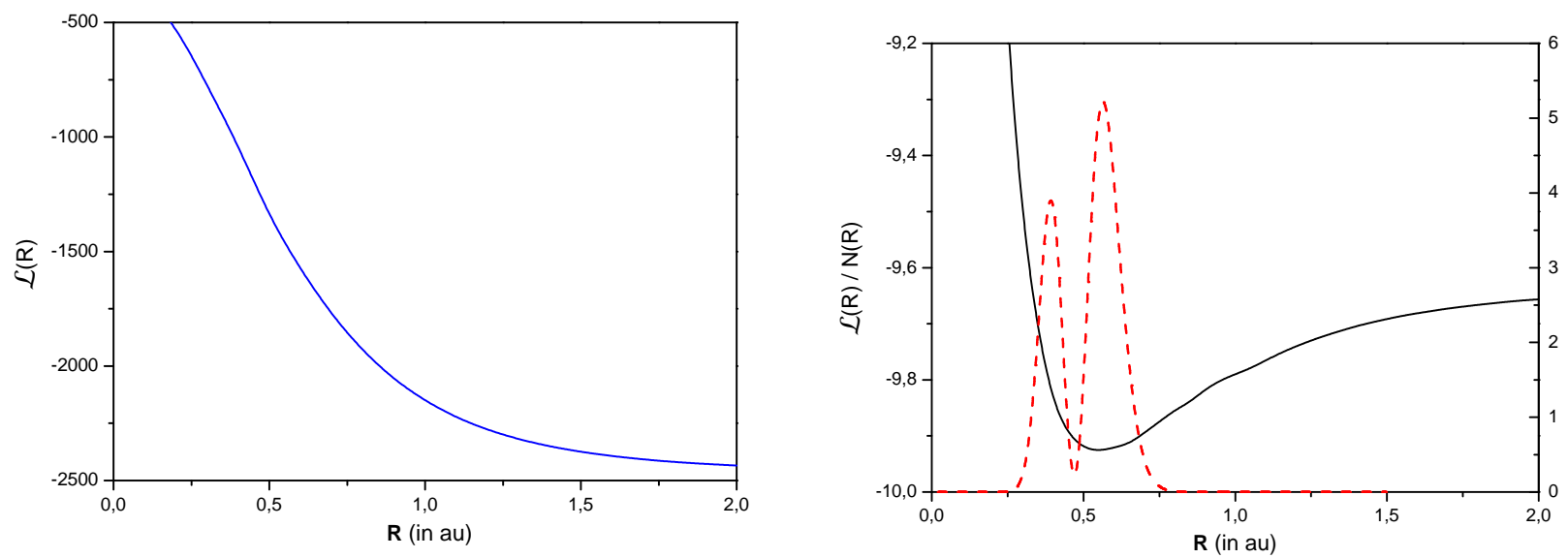

FIG. 7: (Left) The relativistic Bethe logarithm for the ground ( $1 s \sigma)$ electronic state for $Z_{1}=2$ and $Z_{2}=-1$. (Right) The same data, but normalized by the delta function distribution: $N(R)=4 \pi\left(Z_{1}^{3} \delta\left(\mathbf{r}_{1}\right)+Z_{2}^{3} \delta\left(\mathbf{r}_{2}\right)\right)$. The dashed line is the vibrational wave function for the $(36,34)$ state of ${ }^{4} \mathrm{He}^{+} \bar{p}$.

Here the quadrupole operator is defined as

$$
S_{i j}^{(2)}=\frac{1}{2}\left[r_{i} p_{j}+r_{j} p_{i}-\frac{2}{3} \delta_{i j}(\mathbf{r} \cdot \mathbf{p})\right]
$$

and its standard components are

$$
\begin{aligned}
& S_{0}^{(2)}=S_{z z}^{(2)}=\frac{1}{3}\left(2 z p_{z}-x p_{x}-y p_{y}\right) \\
& S_{ \pm 1}^{(2)}=\mp \sqrt{\frac{2}{3}}\left(S_{x z}^{(2)} \pm i S_{y z}^{(2)}\right)=\mp \frac{1}{\sqrt{6}}\left[x p_{z}+z p_{x} \pm i\left(y p_{z}+z p_{y}\right)\right] \\
& S_{ \pm 2}^{(2)}=\frac{1}{\sqrt{6}}\left(S_{x x}^{(2)}-S_{y y}^{(2)} \pm 2 i S_{x y}^{(2)}\right)=\frac{1}{\sqrt{6}}\left[x p_{x}-y p_{y} \pm i\left(x p_{y}+y p_{x}\right)\right]
\end{aligned}
$$

Matrix elements can be computed from the relations (valid for a $\sigma$ state):

$$
\begin{aligned}
& S_{0}^{(2)}=\frac{1}{3}\left(2 z p_{z}+r_{+} p_{-}+r_{-} p_{+}\right)=-\frac{i}{3}\left[2 z\left[\left(z+\frac{R}{2}\right) \frac{1}{r_{1}} \partial_{r_{1}}+\left(z-\frac{R}{2}\right) \frac{1}{r_{2}} \partial_{r_{2}}\right]-r^{2}\left(\frac{1}{r_{1}} \partial_{r_{1}}+\frac{1}{r_{2}} \partial_{r_{2}}\right)\right] \\
& S_{ \pm 1}^{(2)}=\frac{1}{\sqrt{3}}\left(r_{ \pm} p_{z}+z p_{ \pm}\right)= \pm \frac{i}{\sqrt{6}} r e^{ \pm i \varphi}\left(\left(2 z+\frac{R}{2}\right) \frac{1}{r_{1}} \partial_{r_{1}}+\left(2 z-\frac{R}{2}\right) \frac{1}{r_{2}} \partial_{r_{2}},\right) \\
& S_{ \pm 2}^{(2)}=\frac{2}{\sqrt{6}} r_{ \pm} p_{ \pm}=-\frac{i}{\sqrt{6}} r^{2} e^{ \pm i 2 \phi}\left(\frac{1}{r_{1}} \partial_{r_{1}}+\frac{1}{r_{2}} \partial_{r_{2}}\right) .
\end{aligned}
$$

Here $r_{ \pm}$and $p_{ \pm}$are standard components of vector operators $\mathbf{r}$ and $\mathbf{p}$, respectively.

Finally, the matrix elements of the $[\mathbf{r} \times \mathbf{p}]$ operator can be obtained from

$$
[\mathbf{r} \times \mathbf{p}]_{ \pm 1}=\frac{i r e^{ \pm i \phi}}{\sqrt{2}} \frac{R}{2}\left(\frac{1}{r_{1}} \partial_{r_{1}}-\frac{1}{r_{2}} \partial_{r_{2}}\right)
$$

4. In the expression of $P_{r c}^{(2)}(k)$ in Eq. 13, the two terms can be respectively written as

$$
-2\left\langle\left(E_{0}-V\right) p^{i}\left(E_{0}-H-k\right)^{-1} p^{i}\right\rangle
$$

and

$$
\frac{1}{2}\left\langle\left(Z_{1} \frac{\left[\boldsymbol{\sigma} \times \mathbf{r}_{1}\right]^{i}}{r_{1}^{3}}+Z_{2} \frac{\left[\boldsymbol{\sigma} \times \mathbf{r}_{2}\right]^{i}}{r_{2}^{3}}\right)\left(E_{0}-H-k\right)^{-1} p^{i}\right\rangle .
$$




\begin{tabular}{crrrrr}
\hline \hline$R$ & $\beta_{1}$ & $\beta_{2}$ & $\beta_{3}$ & $\mathcal{L}$ & $\mathcal{L} / N(R)$ \\
\hline 0.20 & 29.65 & -584.1 & 62.514 & -491.9 & -8.647 \\
0.25 & 17.558 & -799.9 & 159.373 & -622.9 & -9.1887 \\
0.30 & 1.741 & -998.9 & 236.611 & -760.5 & -9.5198 \\
0.35 & -15.627 & -1191.9 & 305.525 & -902.0 & -9.7143 \\
0.40 & -33.158 & -1382.7 & 369.822 & -1046.0 & -9.8402 \\
0.45 & -49.903 & -1566.8 & 430.590 & -1186.1 & -9.8973 \\
0.50 & -65.195 & -1743.3 & 487.854 & -1320.6 & -9.9213 \\
0.55 & -78.929 & -1909.9 & 541.306 & -1447.5 & -9.9272 \\
0.60 & -91.000 & -2064.5 & 590.636 & -1564.8 & -9.9209 \\
0.65 & -101.172 & -2207.3 & 635.664 & -1672.8 & -9.9137 \\
0.70 & -109.370 & -2335.7 & 676.345 & -1768.7 & -9.8946 \\
0.75 & -116.075 & -2451.0 & 712.787 & -1854.2 & -9.8745 \\
0.80 & -121.547 & -2553.7 & 745.209 & -1930.0 & -9.8546 \\
0.85 & -125.908 & -2645.8 & 773.901 & -1997.8 & -9.8408 \\
0.90 & -127.846 & -2727.0 & 799.189 & -2055.6 & -9.8199 \\
0.95 & -130.235 & -2797.5 & 821.408 & -2106.3 & -9.8013 \\
1.00 & -131.770 & -2860.8 & 840.902 & -2151.6 & -9.7898 \\
1.10 & -133.766 & -2963.8 & 872.905 & -2224.6 & -9.7653 \\
1.20 & -134.563 & -3041.6 & 897.365 & -2278.7 & -9.7402 \\
1.30 & -134.679 & -3101.3 & 916.090 & -2319.8 & -9.7204 \\
1.40 & -134.430 & -3147.1 & 930.468 & -2351.0 & -9.7042 \\
1.50 & -134.006 & -3182.5 & 941.556 & -2374.9 & -9.6915 \\
1.60 & -133.517 & -3209.9 & 950.148 & -2393.2 & -9.6810 \\
1.70 & -133.025 & -3231.3 & 956.847 & -2407.4 & -9.6727 \\
1.80 & -132.561 & -3248.2 & 962.102 & -2418.6 & -9.6664 \\
1.90 & -132.140 & -3261.4 & 966.255 & -2427.2 & -9.6607 \\
2.00 & -131.766 & -3272.0 & 969.562 & -2434.2 & -9.6565 \\
\hline \hline
\end{tabular}

TABLE II: Relativistic Bethe logarithm for the ground $(1 s \sigma)$ electronic state, for $Z_{1}=2$ and $Z_{2}=-1$.

We now show that the second term does not contribute here. Since right-hand transition does not change spin, then on the left-hand side we may keep only those terms, which contain $\sigma_{z}$ :

$$
\mp \frac{i}{2}\left\langle\sigma_{z} \frac{r e^{ \pm i \varphi}}{\sqrt{2}}\left(\frac{Z_{1}}{r_{1}^{3}}+\frac{Z_{2}}{r_{2}^{3}}\right)\left(E_{0}-H-k\right)^{-1} p^{i}\right\rangle
$$

This contribution results in fine splitting of the main line, which is spin-dependent, and thus will not be considered here.

\section{RESULTS}

\section{A. Nonrelativistic Bethe logarithm}

First we perform calculations of the nonrelativistic Bethe logarithm for the two-center Coulomb problem for two cases: $Z_{1}=Z_{2}=1$ and $Z_{1}=2, Z_{2}=-1$. Results are plotted in Fig. 4 . The numerical data for $\beta_{n r}(R)$ is as accurate as 10 significant digits and has been obtained for a large range of internuclear bond lengths $R=0-7$ a.u. with a step of 0.05 a.u. For the hydrogen molecular ion our results are in a good agreement with previous calculations [25]. The complete Tables are too lengthy to be reported here and may be found in the Supplemental Material [26].

\section{B. Relativistic corrections to the Bethe logarithm}

The numerical scheme has been tested on the ground state of the hydrogen atom. Our results are

$$
\begin{aligned}
& \beta_{1}(1 s)=-3.26821319 \\
& \beta_{2}(1 s)=-40.647026693 . \\
& \beta_{3}(1 s)=16.655330436,
\end{aligned}
$$


They are in perfect agreement with the ones of $[12$.

The main results of this work are presented in Tables $\mathrm{I}$ and II Particular behaviour of different components of the relativistic Bethe logarithm $\mathcal{L}$, namely $\beta_{1}^{(a)}, \beta_{1}^{(b)}, \beta_{2}, \beta_{3}$, for a case of a hydrogen molecular ion are shown on Fig. 5 . On Figures 6 and 7 our final results for the low energy contribution $\mathcal{L}(R)$ are plotted.

The numerical calculations have been performed in multiple precision arithmetic with 64 decimal digits. Special care has been required for the nonrelativistic quadrupole contribution at small $R$, in this case a 96 decimal digit arithmetic has been used.

It is worth noting that in the region where the wave function is essentially nonzero the quantity $\mathcal{L} / N(R)$ is about constant: $\mathcal{L} / N(R) \approx 6.5$ for $Z_{1}=Z_{2}=1$ and $\mathcal{L} / N(R) \approx 9.7$ for $Z_{1}=2, Z_{2}=-1$. That may help in a qualitative estimate within 2-3 digit accuracy of the one-loop self-energy contribution to the transition frequencies of not only hydrogen molecular ions but also of the neutral hydrogen molecule $\mathrm{H}_{2}$.

In conclusion, we have computed the low-energy part of the $m \alpha^{7}$ order self-energy correction for the two center problem, with a numerical accuracy that exceeds 3 significant digits, in the whole range of internuclear distances $R \in[0.2,7]$. Calculation of the high energy part is under consideration now. A complete result then should be averaged over vibrational wave functions to get proper correction for ro-vibrational transition frequencies at the $m \alpha^{7}$ order. Taking into account as well the vacuum polarization and two-loop electron self-energy contributions at this order we expect that a relative precision for transition frequencies will be at a level of $10^{-10}$ or better both for hydrogen molecular ions and for antiprotonic helium.

\section{ACKNOWLEDGEMENTS}

The authors are in debt to Krzysztof Pachucki for helpful discussions. V.I.K. acknowledges support of the Russian Foundation for Basic Research under Grant No. 12-02-00417-a. This work was supported by École Normale Supérieure, which is gratefully acknowledged.

\section{Appendix: Two-center matrix elements involving $\ln r_{1}$ or $\ln r_{2}$}

In this Appendix we want to consider an analytical evaluation of the two-center integrals of type [22], which contain either $\ln r_{1}$ or $\ln r_{2}$ as a multiplier. We start from the basic integral:

$$
\Gamma_{\ln r_{1}, 00}(\alpha, \beta)=\int d r_{1} d r_{2}\left[\ln r_{1} \cdot e^{-\alpha r_{1}-\beta r_{2}}\right]
$$

Using identities

$$
\int_{R}^{\infty} d r \ln r e^{-\gamma r}=\frac{1}{\gamma}\left[\mathrm{E}_{1}(R \gamma)+\ln R \cdot e^{-\gamma R}\right]
$$

and

$$
\int_{0}^{R} d r \ln r e^{-\gamma r}=-\frac{1}{\gamma}\left[\mathrm{E}_{1}(R \gamma)+\ln \gamma+\ln R \cdot e^{-\gamma R}+\gamma_{E}\right]
$$

one may get

$$
\begin{aligned}
\int_{R}^{\infty} d r_{1} \ln r_{1} e^{-\alpha r_{1}} \int_{r_{1}-R}^{r_{1}+R} d r_{2} e^{-\beta r_{2}}= \\
=\frac{1}{\beta} \int_{R}^{\infty} d r_{1}\left[\ln r_{1} e^{-\alpha r_{1}}\left(e^{-\beta\left(r_{1}-R\right)}-e^{-\beta\left(r_{1}+R\right)}\right)\right] \\
=\frac{e^{\beta R}-e^{-\beta R}}{\beta} \times \frac{\mathrm{E}_{1}(R(\alpha+\beta))+\ln R \cdot e^{-(\alpha+\beta) R}}{\alpha+\beta}
\end{aligned}
$$


and

$$
\begin{aligned}
\int_{0}^{R} d r_{1} \ln r_{1} e^{-\alpha r_{1}} \int_{R-r_{1}}^{R+r_{1}} d r_{2} e^{-\beta r_{2}}= \\
=\frac{1}{\beta} \int_{0}^{R} d r_{1}\left[\ln r_{1} e^{-\alpha r_{1}}\left(e^{-\beta\left(R-r_{1}\right)}-e^{-\beta\left(R+r_{1}\right)}\right)\right] \\
=\frac{e^{-\beta R}}{\beta}\left[\int_{0}^{R} d r_{1} \ln r_{1} e^{-(\alpha-\beta) r_{1}}-\int_{0}^{R} d r_{1} \ln r_{1} e^{-(\alpha+\beta) r_{1}}\right] \\
=\frac{e^{-\beta R}}{\beta} \times\left[\frac{\mathrm{E}_{1}(R(\alpha+\beta))+\ln (\alpha+\beta)+\ln R \cdot e^{-(\alpha+\beta) R}+\gamma_{E}}{\alpha+\beta}\right. \\
\left.\quad-\frac{\mathrm{E}_{1}(R(\alpha-\beta))+\ln (\alpha-\beta)+\ln R \cdot e^{-(\alpha-\beta) R}+\gamma_{E}}{\alpha-\beta}\right]
\end{aligned}
$$

Summing up the two contributions we obtain a final expression

$$
\begin{aligned}
\Gamma_{\ln r_{1}, 00}(\alpha, \beta)=-\frac{4 \pi}{R} \frac{e^{-\beta R} \gamma_{E}+e^{-\alpha R} \ln R}{\alpha^{2}-\beta^{2}} & +\frac{2 \pi}{R \beta} \frac{e^{\beta R} \mathrm{E}_{1}(R(\alpha+\beta))+e^{-\beta R} \ln (\alpha+\beta)}{\alpha+\beta} \\
& -\frac{2 \pi}{R \beta} \frac{e^{-\beta R}\left[\mathrm{E}_{1}(R(\alpha-\beta))+\ln (\alpha-\beta)\right]}{\alpha-\beta}
\end{aligned}
$$

To generate other integrals one may use

$$
\Gamma_{\ln r_{1}, k l}(\alpha, \beta)=\left(-\frac{\partial}{\partial \alpha}\right)^{k}\left(-\frac{\partial}{\partial \beta}\right)^{l} \Gamma_{\ln r_{1}, 00}(\alpha, \beta) .
$$

[1] J.C.J. Koelemeij, B. Roth, A. Wicht, I. Ernsting, and S. Schiller, Phys. Rev. Lett. 98, 173002 (2007); U. Bressel, A. Borodin, J. Shen, M. Hansen, I. Ernsting, and S. Schiller, Phys. Rev. Lett. 108, 183003 (2012); J. Shen, A. Borodin, M. Hansen, and S. Schiller, Phys. Rev. A 85, 032519 (2012).

[2] J.C.J. Koelemeij, D.W.E. Noom , D. de Jong, M.A. Haddad, and W. Ubachs, Appl. Phys. B 107, 1075 (2012).

[3] J.-Ph. Karr, A. Douillet, and L. Hilico, Appl. Phys. B 107, 1043 (2012).

[4] M. Hori, A. Sótér, D. Barna, A. Dax, R. Hayano, S. Friedreich, B. Juhász, Th. Pask, E. Widmann, D. Horváth, L. Venturelli, and N. Zurlo, Nature 475, 484 (2011).

[5] V.I. Korobov, Phys. Rev. A 77, 022509 (2008).

[6] V.I. Korobov, Phys. Rev. A 77, 042506 (2008).

[7] M.I. Eides, H. Grotch, and V.A. Shelyuto, Theory of Light Hydrogenic Bound States, Springer Tracks in Modern Physics 222, Springer-Verlag, Berlin, Heidelberg (2007).

[8] J.R. Sapirstein, D.R. Yennie, in: T. Kinoshita (Ed.), Quantum Electrodynamics, World Scientific, Singapore, 1990.

[9] K. Pachucki, Phys. Rev. A 46, 648 (1992); K Pachucki, Ann. Phys. (N.Y.) 226, 1 (1993).

[10] U.D. Jentschura, E.-O. Le Bigot, P.J. Mohr, P. Indelicato, and G. Soff, Phys. Rev. Lett. 90, 163001 (2003).

[11] A. Czarnecki, U.D. Jentschura, and K. Pachucki, Phys. Rev. Lett. 95, 180404 (2005).

[12] U.D. Jentschura, A. Czarnecki, and K. Pachucki, Phys. Rev. A 72, 062102 (2005).

[13] W.E. Caswell and J.P. Lepage, Phys. Lett. B 167, 437 (1986).

[14] T. Kinoshita and M. Nio, Phys. Rev. D 53, 4909 (1996).

[15] K. Pachucki, J. Phys. B 31, 3547 (1998).

[16] H.A. Bethe, Phys. Rev. 72, 339 (1947).

[17] H.A. Bethe and E.E. Salpeter, Quantum mechanics of one- and two-electron atoms, Plenum Publishing Co., New York, 1977.

[18] V.I. Korobov, L. Hilico, and J.-Ph. Karr, Phys. Rev. A 79, 012501 (2009).

[19] C. Schwartz, Phys. Rev. 123, 1700 (1961).

[20] V.I. Korobov, Phys. Rev. A 85, 042514 (2012).

[21] M. Gavrila and A. Costescu, Phys. Rev. A 2, 1752 (1970).

[22] Ts. Tsogbayar and V.I. Korobov, J. Chem. Phys. 125, 024308 (2006).

[23] V.I. Korobov, Phys. Rev. A 61, 064503 (2000).

[24] V.I. Korobov and Ts. Tsogbayar, J. Phys. B 80, 2661 (2007).

[25] R. Bukowski, B. Jeziorski, R. Moszyński, and W. Kołos, Int. J. Quantum Chem. 42, 287 (1992).

[26] See Supplemental Material below for complete Tables of the numerical data. 


\title{
Calculation of the relativistic Bethe logarithm in the two-center problem Supplemental Material
}

\author{
Vladimir I. Korobov \\ Bogolyubov Laboratory of Theoretical Physics, Joint Institute for Nuclear Research, \\ Dubna 141980, Russia \\ L. Hilico, and J.-Ph. Karr \\ Laboratoire Kastler Brossel, UPMC-Paris-6, ENS, CNRS \\ Case 74, 4 place Jussieu, 75005 Paris, France
}

In this Supplemental Material, we give the nonrelativistic Bethe logarithm (Table I) and more extensive results for its relativistic corrections (Tables II and III), both for the ground electronic state of $\mathrm{H}_{2}^{+}$and $\mathrm{He}^{+} \bar{p}$. These additional data are helpful for computational use to perform more accurately averaging over vibrational wave functions.

\section{Nonrelativistic Bethe logarithm}

TABLE III: The nonrelativistic Bethe logarithm, $\beta_{n r}$, for $\mathrm{H}_{2}^{+}$and $\mathrm{He}^{+} \bar{p}$.

\begin{tabular}{ccc}
\hline \hline$R$ (in a.u.) & $Z_{1}=1, Z_{2}=1$ & $Z_{1}=2, Z_{2}=-1$ \\
\hline 0.00 & 4.370422917 & 2.984128556 \\
0.05 & 3.960959074 & 4.429093491 \\
0.10 & 3.763208049 & 4.695144972 \\
0.15 & 3.627181789 & 4.737059502 \\
0.20 & 3.525244876 & 4.712352798 \\
0.25 & 3.445386029 & 4.668731134 \\
0.30 & 3.381060204 & 4.622934909 \\
0.35 & 3.328241427 & 4.580920363 \\
0.40 & 3.284256226 & 4.544480350 \\
0.45 & 3.247231763 & 4.513745134 \\
0.50 & 3.215803070 & 4.488209081 \\
0.55 & 3.188944303 & 4.467169500 \\
0.60 & 3.165865517 & 4.449913692 \\
0.65 & 3.145946182 & 4.435793560 \\
0.70 & 3.128690731 & 4.424249332 \\
0.75 & 3.113697833 & 4.414810709 \\
0.80 & 3.100638579 & 4.407088769 \\
0.85 & 3.089240633 & 4.400764849 \\
0.90 & 3.079276477 & 4.395579241 \\
0.95 & 3.070554542 & 4.391320901 \\
1.00 & 3.062912414 & 4.387818567 \\
1.05 & 3.056211557 & 4.384933303 \\
1.10 & 3.050333156 & 4.382552371 \\
1.15 & 3.045174825 & 4.380584212 \\
1.20 & 3.040647951 & 4.378954395 \\
1.25 & 3.036675548 & 4.377602338 \\
1.30 & 3.033190500 & 4.376478663 \\
1.35 & 3.030134120 & 4.375543070 \\
1.40 & 3.027454949 & 4.374762617 \\
1.45 & 3.025107756 & 4.374110337 \\
1.50 & 3.023052703 & 4.373564122 \\
1.55 & 3.021254630 & 4.373105821 \\
1.60 & 3.019682462 & 4.372720508 \\
1.65 & 3.018308688 & 4.372395891 \\
1.70 & 3.017108931 & 4.372121836 \\
1.75 & 3.016061565 & 4.371889968 \\
1.80 & 3.015147393 & 4.371693363 \\
1.85 & 3.014349362 & 4.371526283 \\
1.90 & 3.013652319 & 4.371383970 \\
1.95 & 3.013042797 & 4.371262469 \\
\hline \hline & & \\
\hline
\end{tabular}


TABLE III: (continued)

\begin{tabular}{|c|c|c|}
\hline$R$ (in a.u.) & $Z_{1}=1, Z_{2}=1$ & $Z_{1}=2, Z_{2}=-1$ \\
\hline 2.00 & 3.012508830 & 4.371158489 \\
\hline 2.05 & 3.012039785 & 4.371069288 \\
\hline 2.10 & 3.011626220 & 4.370992576 \\
\hline 2.15 & 3.011259757 & 4.370926439 \\
\hline 2.20 & 3.010932968 & 4.370869276 \\
\hline 2.25 & 3.010639275 & 4.370819742 \\
\hline 2.30 & 3.010372862 & 4.370776708 \\
\hline 2.35 & 3.010128599 & 4.370739223 \\
\hline 2.40 & 3.009901965 & 4.370706488 \\
\hline 2.45 & 3.009688991 & 4.370677824 \\
\hline 2.50 & 3.009486203 & 4.370652660 \\
\hline 2.55 & 3.009290569 & 4.370630511 \\
\hline 2.60 & 3.009099455 & 4.370610966 \\
\hline 2.65 & 3.008910587 & 4.370593673 \\
\hline 2.70 & 3.008722010 & 4.370578334 \\
\hline 2.75 & 3.008532059 & 4.370564694 \\
\hline 2.80 & 3.008339327 & 4.370552535 \\
\hline 2.85 & 3.008142638 & 4.370541670 \\
\hline 2.90 & 3.007941025 & 4.370531937 \\
\hline 2.95 & 3.007733705 & 4.370523199 \\
\hline 3.00 & 3.007520064 & 4.370515335 \\
\hline 3.05 & 3.007299631 & 4.370508243 \\
\hline 3.10 & 3.007072071 & 4.370501832 \\
\hline 3.15 & 3.006837162 & 4.370496026 \\
\hline 3.20 & 3.006594789 & 4.370490756 \\
\hline 3.25 & 3.006344924 & 4.370485963 \\
\hline 3.30 & 3.006087623 & 4.370481595 \\
\hline 3.35 & 3.005823009 & 4.370477609 \\
\hline 3.40 & 3.005551267 & 4.370473962 \\
\hline 3.45 & 3.005272634 & 4.370470622 \\
\hline 3.50 & 3.004987392 & 4.370467557 \\
\hline 3.55 & 3.004695862 & 4.370464739 \\
\hline 3.60 & 3.004398396 & 4.370462145 \\
\hline 3.65 & 3.004095374 & 4.370459753 \\
\hline 3.70 & 3.003787194 & 4.370457545 \\
\hline 3.75 & 3.003474273 & 4.370455503 \\
\hline 3.80 & 3.003157041 & 4.370453612 \\
\hline 3.85 & 3.002835935 & 4.370451859 \\
\hline 3.90 & 3.002511398 & 4.370450231 \\
\hline 3.95 & 3.002183876 & 4.370448719 \\
\hline 4.00 & 3.001853814 & 4.370447311 \\
\hline 4.05 & 3.001521655 & 4.370445999 \\
\hline 4.10 & 3.001187836 & 4.370444776 \\
\hline 4.15 & 3.000852787 & 4.370443634 \\
\hline 4.20 & 3.000516930 & 4.370442567 \\
\hline 4.25 & 3.000180678 & 4.370441568 \\
\hline 4.30 & 2.999844431 & 4.370440633 \\
\hline 4.35 & 2.999508578 & 4.370439757 \\
\hline 4.40 & 2.999173494 & 4.370438934 \\
\hline 4.45 & 2.998839540 & 4.370438162 \\
\hline 4.50 & 2.998507062 & 4.370437437 \\
\hline 4.55 & 2.998176392 & 4.370436754 \\
\hline 4.60 & 2.997847844 & 4.370436112 \\
\hline 4.65 & 2.997521719 & 4.370435506 \\
\hline 4.70 & 2.997198299 & 4.370434936 \\
\hline 4.75 & 2.996877852 & 4.370434397 \\
\hline 4.80 & 2.996560629 & 4.370433889 \\
\hline 4.85 & 2.996246864 & 4.370433409 \\
\hline 4.90 & 2.995936776 & 4.370432955 \\
\hline 4.95 & 2.995630567 & 4.370432525 \\
\hline
\end{tabular}


TABLE III: (continued)

\begin{tabular}{ccc}
\hline \hline$R$ (in a.u.) & $Z_{1}=1, Z_{2}=1$ & $Z_{1}=2, Z_{2}=-1$ \\
\hline 5.00 & 2.995328425 & 4.370432118 \\
5.05 & 2.995030520 & 4.370431733 \\
5.10 & 2.994737010 & 4.370431368 \\
5.15 & 2.994448035 & 4.370431021 \\
5.20 & 2.994163724 & 4.370430693 \\
5.25 & 2.993884189 & 4.370430380 \\
5.30 & 2.993609531 & 4.370430084 \\
5.35 & 2.993339837 & 4.370429802 \\
5.40 & 2.993075180 & 4.370429533 \\
5.45 & 2.992815623 & 4.370429278 \\
5.50 & 2.992561218 & 4.370429035 \\
5.55 & 2.992312003 & 4.370428803 \\
5.60 & 2.992068009 & 4.370428583 \\
5.65 & 2.991829253 & 4.370428372 \\
5.70 & 2.991595748 & 4.370428171 \\
5.75 & 2.991367492 & 4.370427980 \\
5.80 & 2.991144480 & 4.370427797 \\
5.85 & 2.990926695 & 4.370427622 \\
5.90 & 2.990714115 & 4.370427455 \\
5.95 & 2.990506709 & 4.370427295 \\
6.00 & 2.990304442 & 4.370427142 \\
6.05 & 2.990107272 & 4.370426996 \\
6.10 & 2.989915150 & 4.370426856 \\
6.15 & 2.989728023 & 4.370426722 \\
6.20 & 2.989545835 & 4.370426593 \\
6.25 & 2.989368523 & 4.370426470 \\
6.30 & 2.989196022 & 4.370426352 \\
6.35 & 2.989028262 & 4.370426239 \\
6.40 & 2.988865171 & 4.370426130 \\
6.45 & 2.988706674 & 4.370426025 \\
6.50 & 2.988552692 & 4.370425925 \\
6.55 & 2.988403148 & 4.370425829 \\
6.60 & 2.988257957 & 4.370425736 \\
6.65 & 2.988117039 & 4.370425647 \\
6.70 & 2.987980307 & 4.370425562 \\
6.75 & 2.987847676 & 4.370425479 \\
6.80 & 2.987719061 & 4.370425400 \\
6.85 & 2.987594373 & 4.370425324 \\
6.90 & 2.987473527 & 4.370425251 \\
6.95 & 2.987356433 & 4.370425180 \\
7.00 & 2.987243004 & 4.370425112 \\
\hline \hline & & \\
\hline
\end{tabular}

Relativistic correction to the Bethe logarithm

TABLE IV: Relativistic Bethe logarithm for the ground $\left(1 s \sigma_{g}\right)$ electronic state for $Z_{1}=Z_{2}=1$.

\begin{tabular}{ccccccc}
\hline \hline$R$ & $\beta_{1}^{(a)}$ & $\beta_{1}^{(b)}$ & $\beta_{2}$ & \multicolumn{1}{c}{$\beta_{3}$} & \multicolumn{1}{c}{$\mathcal{L}$} & $\mathcal{L} / N(R)$ \\
\hline 0.2 & 199.124 & -362.792 & -718.74 & 500.9 & -381.5 & -9.4774 \\
0.3 & 71.425 & -155.864 & -408.41 & 244.09 & -248.76 & -7.7149 \\
0.4 & 26.866 & -73.891 & -293.55 & 153.814 & -186.76 & -7.0981 \\
0.5 & 8.5247 & -36.4368 & -232.469 & 111.218 & -149.163 & -6.8191 \\
0.6 & 0.3419 & -17.8754 & -192.985 & 86.9905 & -123.529 & -6.6763 \\
0.7 & -3.4173 & -8.19603 & -164.670 & 71.3995 & -104.884 & -6.5980 \\
0.8 & -5.1044 & -2.99381 & -143.164 & 60.5041 & -90.7581 & -6.5534 \\
0.9 & -5.7669 & -0.16341 & -126.255 & 52.4484 & -79.7371 & -6.5273 \\
1.0 & -5.94144 & 1.36042 & -112.653 & 46.2540 & -70.9802 & -6.5145 \\
1.1 & -5.85242 & 2.14699 & -101.528 & 41.3527 & -63.8807 & -6.5084 \\
\hline \hline
\end{tabular}


TABLE IV: (continued)

\begin{tabular}{|c|c|c|c|c|c|c|}
\hline$R$ & $\beta_{1}^{(a)}$ & $\beta_{1}^{(b)}$ & $\beta_{2}$ & $\beta_{3}$ & $\mathcal{L}$ & $\mathcal{L} / N(R)$ \\
\hline 1.2 & -5.64166 & 2.50981 & -92.3095 & 37.3908 & -58.0506 & -6.5071 \\
\hline 1.3 & -5.37875 & 2.62712 & -84.5938 & 34.1346 & -53.2109 & -6.5097 \\
\hline 1.4 & -5.10067 & 2.60269 & -78.0750 & 31.4227 & -49.1503 & -6.5145 \\
\hline 1.5 & -4.82677 & 2.49792 & -72.5270 & 29.1400 & -45.7159 & -6.5212 \\
\hline 1.6 & -4.56684 & 2.34961 & -67.7744 & 27.2013 & -42.7903 & -6.5294 \\
\hline 1.7 & -4.32531 & 2.17994 & -63.6799 & 25.5425 & -40.2828 & -6.5387 \\
\hline 1.8 & -4.10371 & 2.00222 & -60.1351 & 24.1145 & -38.1221 & -6.5489 \\
\hline 1.9 & -3.90197 & 1.82441 & -57.0531 & 22.8786 & -36.2520 & -6.5598 \\
\hline 2.0 & -3.71921 & 1.65108 & -54.3637 & 21.8045 & -34.6274 & -6.5712 \\
\hline 2.1 & -3.55413 & 1.48479 & -52.0099 & 20.8676 & -33.2117 & -6.5831 \\
\hline 2.2 & -3.40532 & 1.32679 & -49.9446 & 20.0480 & -31.9752 & -6.5953 \\
\hline 2.3 & -3.27133 & 1.17755 & -48.1290 & 19.3294 & -30.8933 & -6.6077 \\
\hline 2.4 & -3.15078 & 1.03708 & -46.5306 & 18.6985 & -29.9458 & -6.6203 \\
\hline 2.5 & -3.04237 & 0.90509 & -45.1223 & 18.1441 & -29.1155 & -6.6329 \\
\hline 2.6 & -2.94493 & 0.78117 & -43.8811 & 17.6566 & -28.3883 & -6.6456 \\
\hline 2.7 & -2.85740 & 0.66481 & -42.7875 & 17.2283 & -27.7518 & -6.6583 \\
\hline 2.8 & -2.77881 & 0.55550 & -41.8248 & 16.8522 & -27.1958 & -6.6709 \\
\hline 2.9 & -2.70829 & 0.45272 & -40.9786 & 16.5527 & -26.6814 & -6.6758 \\
\hline 3.0 & -2.64509 & 0.35594 & -40.2365 & 16.2347 & -26.2909 & -6.6955 \\
\hline 3.1 & -2.58852 & 0.26478 & -39.5878 & 15.9839 & -25.9276 & -6.7075 \\
\hline 3.2 & -2.53795 & 0.17881 & -39.0229 & 15.7664 & -25.6156 & -6.7192 \\
\hline 3.3 & -2.49285 & 0.09764 & -38.5337 & 15.5791 & -25.3498 & -6.7306 \\
\hline 3.4 & -2.45270 & 0.02094 & -38.1126 & 15.4188 & -25.1256 & -6.7416 \\
\hline 3.5 & -2.41708 & -0.05155 & -37.7533 & 15.2831 & -24.9389 & -6.7522 \\
\hline 3.6 & -2.38556 & -0.12013 & -37.4498 & 15.1695 & -24.7860 & -6.7624 \\
\hline 3.7 & -2.35780 & -0.18499 & -37.1970 & 15.0761 & -24.6637 & -6.7722 \\
\hline 3.8 & -2.33346 & -0.24634 & -36.9899 & 15.0008 & -24.5689 & -6.7815 \\
\hline 3.9 & -2.31223 & -0.30433 & -36.8243 & 14.9419 & -24.4990 & -6.7903 \\
\hline 4.0 & -2.29385 & -0.35913 & -36.6963 & 14.8980 & -24.4513 & -6.7985 \\
\hline 4.1 & -2.27805 & -0.41085 & -36.6028 & 14.8674 & -24.4243 & -6.8065 \\
\hline 4.2 & -2.26461 & -0.45960 & -36.5387 & 14.8490 & -24.4139 & -6.8135 \\
\hline 4.3 & -2.25331 & -0.50549 & -36.5027 & 14.8414 & -24.4201 & -6.8202 \\
\hline 4.4 & -2.24395 & -0.54860 & -36.4913 & 14.8435 & -24.4404 & -6.8264 \\
\hline 4.5 & -2.23635 & -0.58902 & -36.5019 & 14.8542 & -24.4730 & -6.8320 \\
\hline 4.6 & -2.23034 & -0.62682 & -36.5320 & 14.8726 & -24.5165 & -6.8370 \\
\hline 4.7 & -2.22576 & -0.66208 & -36.5793 & 14.8978 & -24.5694 & -6.8416 \\
\hline 4.8 & -2.22246 & -0.69487 & -36.6417 & 14.9288 & -24.6303 & -6.8456 \\
\hline 4.9 & -2.22031 & -0.72527 & -36.7172 & 14.9649 & -24.6979 & -6.8492 \\
\hline 5.0 & -2.21917 & -0.75335 & -36.8039 & 15.0053 & -24.7712 & -6.8523 \\
\hline 5.1 & -2.21893 & -0.77921 & -36.9002 & 15.0493 & -24.8490 & -6.8549 \\
\hline 5.2 & -2.21949 & -0.80291 & -37.0044 & 15.0964 & -24.9304 & -6.8571 \\
\hline 5.3 & -2.22073 & -0.82454 & -37.1151 & 15.1459 & -25.0145 & -6.8588 \\
\hline 5.4 & -2.22257 & -0.84421 & -37.2308 & 15.1972 & -25.1004 & -6.8602 \\
\hline 5.5 & -2.22492 & -0.86200 & -37.3505 & 15.2499 & -25.1875 & -6.8612 \\
\hline 5.6 & -2.22770 & -0.87800 & -37.4729 & 15.3036 & -25.2750 & -6.8619 \\
\hline 5.7 & -2.23084 & -0.89231 & -37.5970 & 15.3578 & -25.3624 & -6.8623 \\
\hline 5.8 & -2.23427 & -0.90504 & -37.7220 & 15.4121 & -25.4493 & -6.8624 \\
\hline 5.9 & -2.23794 & -0.91629 & -37.8470 & 15.4662 & -25.5350 & -6.8623 \\
\hline 6.0 & -2.24178 & -0.92615 & -37.9714 & 15.5199 & -25.6194 & -6.8619 \\
\hline 6.1 & -2.24576 & -0.93472 & -38.0943 & 15.5729 & -25.7019 & -6.8613 \\
\hline 6.2 & -2.24982 & -0.94211 & -38.2154 & 15.6250 & -25.7824 & -6.8606 \\
\hline 6.3 & -2.25394 & -0.94839 & -38.3342 & 15.6760 & -25.8606 & -6.8597 \\
\hline 6.4 & -2.25808 & -0.95368 & -38.4501 & 15.7256 & -25.9363 & -6.8587 \\
\hline 6.5 & -2.26220 & -0.95805 & -38.5630 & 15.7739 & -26.0093 & -6.8576 \\
\hline 6.6 & -2.26629 & -0.96158 & -38.6725 & 15.8207 & -26.0797 & -6.8563 \\
\hline 6.7 & -2.27032 & -0.96439 & -38.7784 & 15.8658 & -26.1473 & -6.8551 \\
\hline 6.8 & -2.27427 & -0.96652 & -38.8806 & 15.9094 & -26.2120 & -6.8537 \\
\hline 6.9 & -2.27814 & -0.96805 & -38.9789 & 15.9512 & -26.2739 & -6.8523 \\
\hline 7.0 & -2.28190 & -0.96905 & -39.0732 & 15.9913 & -26.3328 & -6.8509 \\
\hline
\end{tabular}


TABLE V: Relativistic Bethe logarithm for the ground ( $1 s \sigma)$ electronic state, for $Z_{1}=2$ and $Z_{2}=-1$.

\begin{tabular}{cccccc}
\hline \hline$R$ & $\beta_{1}$ & $\beta_{2}$ & $\beta_{3}$ & $\mathcal{L}$ & $\mathcal{L} / N(R)$ \\
\hline 0.10 & 192.1 & -102. & -381.08 & -290.9 & -7.3871 \\
0.15 & 38.01 & -288. & -81.305 & -331.2 & -6.9913 \\
0.20 & 29.65 & -584.1 & 62.514 & -491.9 & -8.6471 \\
0.25 & 17.558 & -799.9 & 159.373 & -622.9 & -9.1887 \\
0.30 & 1.741 & -998.9 & 236.611 & -760.5 & -9.5198 \\
0.35 & -15.627 & -1191.9 & 305.525 & -902.0 & -9.7143 \\
0.40 & -33.158 & -1382.7 & 369.822 & -1046.0 & -9.8402 \\
0.45 & -49.903 & -1566.8 & 430.590 & -1186.1 & -9.8973 \\
0.50 & -65.195 & -1743.3 & 487.854 & -1320.6 & -9.9213 \\
0.55 & -78.929 & -1909.9 & 541.306 & -1447.5 & -9.9272 \\
0.60 & -91.000 & -2064.5 & 590.636 & -1564.8 & -9.9209 \\
0.65 & -101.172 & -2207.3 & 635.664 & -1672.8 & -9.9137 \\
0.70 & -109.370 & -2335.7 & 676.345 & -1768.7 & -9.8946 \\
0.75 & -116.075 & -2451.0 & 712.787 & -1854.2 & -9.8745 \\
0.80 & -121.547 & -2553.7 & 745.209 & -1930.0 & -9.8546 \\
0.85 & -125.908 & -2645.8 & 773.901 & -1997.8 & -9.8408 \\
0.90 & -127.846 & -2727.0 & 799.189 & -2055.6 & -9.8199 \\
0.95 & -130.235 & -2797.5 & 821.408 & -2106.3 & -9.8013 \\
1.00 & -131.770 & -2860.8 & 840.902 & -2151.6 & -9.7898 \\
1.05 & -132.958 & -2916.3 & 857.985 & -2191.2 & -9.7795 \\
1.10 & -133.766 & -2963.8 & 872.905 & -2224.6 & -9.7653 \\
1.15 & -134.277 & -3005.3 & 885.953 & -2253.6 & -9.7521 \\
1.20 & -134.563 & -3041.6 & 897.365 & -2278.7 & -9.7402 \\
1.25 & -134.682 & -3073.4 & 907.349 & -2300.7 & -9.7297 \\
1.30 & -134.679 & -3101.3 & 916.090 & -2319.8 & -9.7204 \\
1.35 & -134.586 & -3125.7 & 923.750 & -2336.5 & -9.7119 \\
1.40 & -134.430 & -3147.1 & 930.468 & -2351.0 & -9.7042 \\
1.45 & -134.232 & -3165.9 & 936.368 & -2363.7 & -9.6974 \\
1.50 & -134.006 & -3182.5 & 941.556 & -2374.9 & -9.6915 \\
1.55 & -133.765 & -3197.1 & 946.122 & -2384.7 & -9.6861 \\
1.60 & -133.517 & -3209.9 & 950.148 & -2393.2 & -9.6810 \\
1.65 & -133.268 & -3221.3 & 953.703 & -2400.8 & -9.6768 \\
1.70 & -133.025 & -3231.3 & 956.847 & -2407.4 & -9.6727 \\
1.75 & -132.788 & -3240.3 & 959.631 & -2413.4 & -9.6696 \\
1.80 & -132.561 & -3248.2 & 962.102 & -2418.6 & -9.6664 \\
1.85 & -132.344 & -3255.2 & 964.299 & -2423.2 & -9.6635 \\
1.90 & -132.140 & -3261.4 & 966.255 & -2427.2 & -9.6607 \\
1.95 & -131.947 & -3267.0 & 968.001 & -2430.9 & -9.6585 \\
2.00 & -131.766 & -3272.0 & 969.562 & -2434.2 & -9.6565 \\
\hline \hline & & & & &
\end{tabular}

